\title{
Microwave assisted solvent-, support- and catalyst-free synthesis of enaminones
}

\author{
Carlos Kleber Z. Andrade,* Angélica de Fátima S. Barreto, and Wender A. Silva \\ Laboratório de Química Metodológica e Orgânica Sintética (LaQMOS), Instituto de Química, \\ Universidade de Brasília, C.P. 4478, 70904-970, Brasília, DF, Brazil \\ E-mail: ckleber@unb.br
}

\section{Dedicated to Prof. Larry E. Overman on the occasion of his $65^{\text {th }}$ anniversary}

\begin{abstract}
Microwave irradiations of different sources were used to prepare various enaminones from the corresponding free amines and $\beta$-dicarbonyl compounds under solvent-, support- and catalystfree conditions.
\end{abstract}

Keywords: Microwaves, enaminones, solvent-free synthesis

\section{Introduction}

Currently, there has been an increasing demand for efficient synthetic processes and solvent-free reactions due to environmental concerns. Some old and new methodologies are being used to diminish and prevent pollution caused by chemical activities.

In this context, the microwave oven has become an important source of heating in many laboratory procedures. ${ }^{1}$ Microwave irradiation has been frequently used in diverse organic transformations with a remarkable reduction in reaction times and, in many cases, improving the yields and selectivities of the processes. ${ }^{2}$

Furthermore, microwave assisted solvent-free organic synthesis (MASFOS) has been developed as an environmentally friendly process once it puts together the cleanliness associated with most reactions realized under microwaves with a solvent- and waste-free process in which organic solvents are avoided throughout all stages. ${ }^{3}$ In an effort to apply this concept and aiming at a simple and efficient preparation of useful synthetic intermediates, we wish to report here the solvent-free synthesis of several enaminones, under MW irradiation, using the free amines and without the need for solid supports or acid catalysts as described in other methodologies. ${ }^{4}$ Another important point of this work is to demonstrate that in almost all examples shown here there is no significant difference in the results of the reactions carried out in a domestic microwave oven and those carried out in a commercial microwave reactor. 
The versatility of enaminones is in great part due to their promptness to both electrophilic and nucleophilic attack. ${ }^{5}$ For this reason, they have been used in the synthesis of various heterocycles ${ }^{1 \mathrm{e}, 6}$ and natural products ${ }^{7}$ and hence several methodologies have been recently developed towards their synthesis, ${ }^{4}$ representing great achievements compared to the original procedure. ${ }^{8}$ Besides reducing the reaction time and increasing the yield and the efficiency of the process, most of them focus on a cleaner way to obtain enaminones. The use of solid supports, ${ }^{4 a-d}$ ionic liquids ${ }^{4 \mathrm{~d}}$ and different types of catalysts ${ }^{4 \mathrm{e}-\mathrm{n}}$ has been reported as effective.

Domestic microwave irradiation has already been described in the preparation of enaminones by Braibante et al. ${ }^{4 a, b}$ and by Hamelin et al. ${ }^{9}$ In both cases, either a solid support or an acid catalyst was employed as reaction promoter. During the course of our study, Lee et al. ${ }^{40}$ reported a solvent-free microwave assisted preparation of enaminones in a MW reactor, using catalytic $\mathrm{HCl}$ and an excess of the amines.

\section{Results and Discussion}

In the synthesis of enaminones described herein, aliphatic and aromatic amines and a diamine were used in the reactions with an equivalent amount of acetylacetone, ethyl acetoacetate and 1,3-cyclohexadione (Scheme 1 and Figure 1). For the sake of comparison, the reactions were carried out both in a domestic microwave oven (Panasonic Inverter) and in a microwave reactor (CEM Co., Discover) and were monitored by TLC. In the first case, the oven was always turned off after each 3-minute period of heating to cool down the reaction mixture and avoid the evaporation of the reagents.
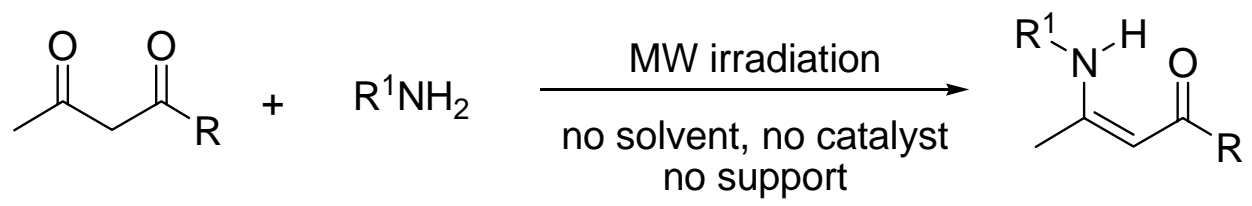

Scheme 1. Synthesis of enaminones under MW irradiation.

Amines substituted with both electron withdrawing and electron donating groups could be succesfully used, except for $p$-nitroaniline which is not soluble in acetylacetone and did not react even at prolonged reaction times or by adding $\mathrm{HCl}$ or under a greater MW potency. As expected, dimeric enaminones were obtained when ethylenediamine was reacted with either acetylacetone or ethyl acetoacetate (Scheme 2). Interestingly, in the first case, the enaminone was not formed using the MW reactor, instead a complex mixture of products was obtained. Cyclic enaminones were also obtained efficiently from 1,3-cyclohexadione. The yields of all reactions were greater than $90 \%$ and the products were obtained in pure form without the need for purification. They were characterized by ${ }^{1} \mathrm{H}$ and ${ }^{13} \mathrm{C}$ NMR, IR and melting points giving data consistent with those 
reported in the literature. It is important to point out that no organic solvent was used at any time in these procedures.

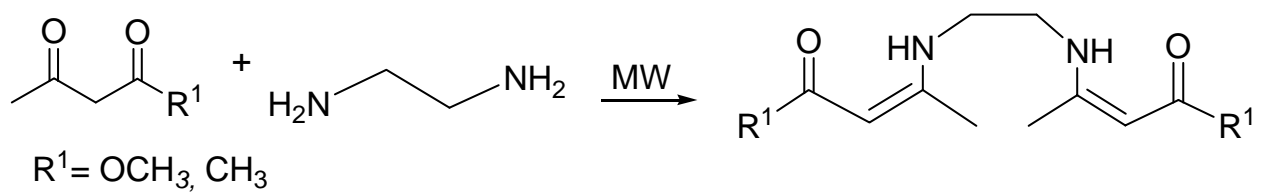

Scheme 2. Formation of dimeric enaminones under MW irradiation.<smiles>CC(=O)/C=C(/C)Nc1ccccc1</smiles>

A: 9 min - 92\%

B: 8 min - 100\%<smiles>CC(=O)/C=C(/C)Nc1ccc(C)cc1</smiles>

A: $12 \mathrm{~min}-91 \%$

B: 12 min - 98\%<smiles>COc1ccc(N/C(C)=C\C(C)=O)cc1</smiles>

A: $32 \mathrm{~min}-96 \%$ B: 26 min - 98\%<smiles>COc1ccccc1N/C(C)=C\C(C)=O</smiles>

A: $40 \mathrm{~min}-91 \%$

B: $26 \min -98 \%$<smiles>CC(=O)/C=C(/C)Nc1ccc(Br)cc1</smiles>

A: $12 \mathrm{~min}-93 \%$ B: 9 min - 100\%<smiles>CC(=O)/C=C(/C)NCCc1ccccc1</smiles>

A: 12 min - 98\%<smiles>[3H]C(C)=CC(C)=CCCC</smiles>

A: $28 \mathrm{~min}-92 \%$

B: 25 min - 92\%<smiles>CCOC(=O)/C=C(\C)NCCN/C(C)=C/C(=O)OCC</smiles>

A: 6 min - $95 \%$

B: 4 min - 95\%<smiles>CC(=O)/C=C(/C)NCCN/C(C)=C/C(C)=O</smiles>

A: 3 min - 96\%

B: 3 min - 0\%<smiles>O=C1C=C(Nc2ccccc2)CCC1</smiles>

A: $6 \min -100 \%$

B: 4 min - 90\%<smiles>Cc1ccccc1NC1=CC(=O)CCC1</smiles>

A: $30 \mathrm{~min}-90 \%$

B: 28 min - 97\%<smiles>COc1ccccc1NC1=CC(=O)CCC1</smiles>

A: 6 min - 97\%

B: 4 min - 98\%<smiles>COc1cccc(NC2=CC(=O)CCC2)c1</smiles>

A: $30 \mathrm{~min}-98 \%$

B: 26 min - 98\%

Method A: domestic MW oven (Panasonic Inverter), $160 \mathrm{~W}$

Method B: MW reactor (CEM Co., Discover), $150-180{ }^{\circ} \mathrm{C}, 150 \mathrm{~W}$

Figure 1. Results of the MW assisted synthesis of enaminones. 
Due to the fact that no catalyst is being used whatsoever, some reactions take a longer time to complete (30-40 min). This time can be reduced by using some drops of concentrated $\mathrm{HCl}$ as described in the work of Lee et al. ${ }^{40}$ For instance, the reaction of $o$-anisidine with acetylacetone in the domestic MW was complete in 10 minutes in a similar yield as compared to 40 minutes in the uncatalysed reaction. Nevertheless, the use of a concentrated acid, even in tiny amounts, is not compatible with a so-called green process.

As already mentioned, there was not a significant difference in the reactions related to the source of microwaves. The yields were slightly better in the MW reactor except for one case. Most reactions were complete within approximately the same time. As discussed before, there was only one case in which the enaminone was not formed using the MW reactor. A control experiment was realized in order to measure the importance of the MW irradiation in these reactions. When $1 \mathrm{mmol}$ of either $o$-toluidine or $o$-anisidine was refluxed with acetylacetone $(1 \mathrm{mmol})$, at $150-165^{\circ} \mathrm{C}$, without solvent, no reaction took place after $8 \mathrm{~h}$ and $6 \mathrm{~h}$, respectively.

In summary, a simple an efficient method was achieved for the preparation of enaminones, employing a green methodology. The simplicity of the reaction conditions, their efficacy and the excellent results obtained using both available forms of MW irradiation, under solvent-, supportand catalyst-free conditions, constitute an attractive contribution among the existing methodologies.

\section{Experimental Section}

General Procedures. Melting points were recorded on a Thomas Hoover Capillary melting point apparatus and are uncorrected. IR spectra were recorded on a Bomem MB-100 FTIR. ${ }^{1} \mathrm{H}$ and ${ }^{13} \mathrm{C}$ NMR spectra were recorded on a Varian Mercury Plus 300 spectrometer, using $\mathrm{CDCl}_{3}$ as solvent and TMS as an internal standard. All compounds were analyzed by ${ }^{1} \mathrm{H} N M R,{ }^{13} \mathrm{C} N M R$, IR and melting points (for solid products) giving data consistent with those published.

\section{General experimental procedures}

Method A. An open $125 \mathrm{~mL}$ Erlenmeyer flask containing the amine $(1 \mathrm{mmol})$ and the dicarbonyl compound $(1 \mathrm{mmol})$ was introduced in a domestic microwave oven (Panasonic Inverter) and irradiated for 3-40 min $(160 \mathrm{~W})$. The oven was turned off always after 3-minute periods of heating to avoid evaporation of the reagents. The reactions were monitored by TLC and after completion the flask was cooled and the products were removed from the flask either by scratching or with a pipette and weighted for yields determination.

Method B. A sealed $10 \mathrm{~mL}$ glass tube containing the amine $(1 \mathrm{mmol})$ and the dicarbonyl compound $(1 \mathrm{mmol})$ was introduced in the cavity of a microwave reactor (CEM Co., Discover) and irradiated for 3-28 min $(150 \mathrm{~W})$ under magnetic stirring. The temperature raised until 150$180{ }^{\circ} \mathrm{C}$. The reactions were monitored by TLC and after completion the glass tube was cooled 
and the products were removed from the tube either by scratching or with a pipette and weighted for yields determination.

\section{Selected spectroscopic data}

4-Phenylamino-pent-3-en-2-one (1). ${ }^{4 \mathrm{j}} \mathrm{mp}, 49-50{ }^{\circ} \mathrm{C}$ (Lit. $\left.{ }^{4 \mathrm{j}} \mathrm{mp}, 48-49{ }^{\circ} \mathrm{C}\right)$; IR $\left(\mathrm{cm}^{-1}\right)$ : $v_{\text {máx }} 2996$, $1615,1573,1509,1281,1191,1019,924,750,695,507 ;{ }^{1} \mathrm{H} \mathrm{NMR}\left(\mathrm{CDCl}_{3}, 300 \mathrm{MHz}\right): \delta 12.49$ (br s, 1H), 7.09-7.36 (m, 5H), $5.19(\mathrm{~s}, 1 \mathrm{H}), 2.10(\mathrm{~s}, 3 \mathrm{H}), 1.99(\mathrm{~s}, 3 \mathrm{H}) ;{ }^{13} \mathrm{C} \mathrm{NMR}\left(\mathrm{CDCl}_{3}, 75\right.$ $\mathrm{MHz}): \delta 195.9,160.0,138.5,128.9,125.4,124.5,97.4,28.9,19.6$.

4-(4-Methyl-phenylamino)-pent-3-en-2-one (2). ${ }^{41} \mathrm{mp}, 58-60{ }^{\circ} \mathrm{C}$ (Lit. ${ }^{41} \mathrm{mp}, 58-59{ }^{\circ} \mathrm{C}$ ); IR $\left(\mathrm{cm}^{-1}\right): v_{\text {máx }} 3443,3026,2997,2923,1609,1566,1520,1283,1017,809 ;{ }^{1} \mathrm{H} \mathrm{NMR}\left(\mathrm{CDCl}_{3}, 300\right.$ MHz): $\delta 12.41($ br s, 1H), 7.27-6.98 (m, 4H), $5.17(\mathrm{~s}, 1 \mathrm{H}) 2.34(\mathrm{~s}, 3 \mathrm{H}), 2.09(\mathrm{~s}, 3 \mathrm{H}), 1.96(\mathrm{~s}, 3 \mathrm{H})$; ${ }^{13} \mathrm{C} \mathrm{NMR}\left(\mathrm{CDCl}_{3}, 75 \mathrm{MHz}\right): \delta 195.5,160.3,135.8,135.1,129.4,124.5,96.9,28.8,20.6,19.4$.

4-(4-Methoxy-phenylamino)-pent-3-en-2-one (3). ${ }^{4 \mathrm{~d}} \mathrm{IR}\left(\mathrm{cm}^{-1}\right)$ 3583, 2999, 2836, 1613, 1569 , 1514, 1439, 1279, 1183, 996, 841, 746; ${ }^{1} \mathrm{H}$ NMR (CDCl $\left.3,300 \mathrm{MHz}\right): \delta 12.29$ (br s, $\left.1 \mathrm{H}\right), 7.04$ (d, $2 \mathrm{H}, J=9.1 \mathrm{~Hz}), 6.90(\mathrm{~d}, 2 \mathrm{H}, J=9.1 \mathrm{~Hz}), 5.16(\mathrm{~s}, 1 \mathrm{H}), 3.80(\mathrm{~s}, 3 \mathrm{H}), 2.09$ (s, 3H), $1.91(\mathrm{~s}, 3 \mathrm{H})$; ${ }^{13} \mathrm{C} \mathrm{NMR}\left(\mathrm{CDCl}_{3}, 75 \mathrm{MHz}\right): \delta$ 195.5, 161.2, 157.5, 131.2, 126.4, 114.0, 96.7, 55.3, 28.8, 19.4.

4-(2-Methoxy-phenylamino)-pentan-2-one (4). ${ }^{41} \mathrm{IR}\left(\mathrm{cm}^{-1}\right)$ 3447, 3069, 3008, 1616, 1571, 1507, 1279, 1175, 1026, 923, 666, 517. ${ }^{1} \mathrm{H} \mathrm{NMR}\left(\mathrm{CDCl}_{3}, 300 \mathrm{MHz}\right): \delta 12.33$ (br s, 1H), 7.26$6.71(\mathrm{~m}, 4 \mathrm{H}), 5.21(\mathrm{~s}, 1 \mathrm{H}), 3.87(\mathrm{~s}, 3 \mathrm{H}), 2.11(\mathrm{~s}, 3 \mathrm{H}), 2.00(\mathrm{~s}, 3 \mathrm{H}) .{ }^{13} \mathrm{C} \mathrm{NMR}\left(\mathrm{CDCl}_{3}, 75 \mathrm{MHz}\right)$ : $\delta 196.4,161.3,154.2,132.4,125.8,114.2,112.3,98.8,77.2,56.4,28.7,19.6$.

4-(4-Bromo-phenylamino)-pent-3-en-2-one (5). ${ }^{4 \mathrm{~m}} \mathrm{mp}, 49-51{ }^{\circ} \mathrm{C}$ (Lit. ${ }^{4 \mathrm{~m}} \mathrm{mp}, 49-50{ }^{\circ} \mathrm{C}$ ); IR $\left(\mathrm{cm}^{-1}\right): v_{\text {máx }} 3455,3068,1613,1563,1507,1277,1024,751,606 .{ }^{1} \mathrm{H} \mathrm{NMR}\left(\mathrm{CDCl}_{3}, 300 \mathrm{MHz}\right)$ : $\delta 12.43($ br s, 1H), $7.44(\mathrm{~d}, 2 \mathrm{H}, J=8.7 \mathrm{~Hz}), 6.98(\mathrm{~d}, 2 \mathrm{H}, J=8.7 \mathrm{~Hz}), 5.21(\mathrm{~s}, 1 \mathrm{H}), 2.10(\mathrm{~s}, 3 \mathrm{H})$, 1.99 (s, 3H); ${ }^{13} \mathrm{C} \mathrm{NMR}\left(\mathrm{CDCl}_{3}, 75 \mathrm{MHz}\right): \delta 196.5,159.5,137.7,132.1,126.0,116.6,98.1,29.1$, 19.7 .

4-(Phenethylamino)pent-3-en-2-one (6). ${ }^{10} \mathrm{IR}\left(\mathrm{cm}^{-1}\right): v_{\text {máx }} 3583,3065,3027,1610,1575,1519$, 1296, 1195, 1030, 932, 749, 666, 518. ${ }^{1} \mathrm{H} \mathrm{NMR}\left(\mathrm{CDCl}_{3}, 300 \mathrm{MHz}\right): \delta 10.89$ (br s, $\left.1 \mathrm{H}\right), 7.36-7.15$ $(\mathrm{m}, 5 \mathrm{H}), 4.93(\mathrm{~s}, 1 \mathrm{H}), 3.53-3.40(\mathrm{~m}, 2 \mathrm{H}), 2.96-2.74(\mathrm{~m}, 2 \mathrm{H}), 1.99(\mathrm{~s}, 3 \mathrm{H}), 1.79(\mathrm{~s}, 3 \mathrm{H}) .{ }^{13} \mathrm{C}$ NMR $\left(\mathrm{CDCl}_{3}, 75 \mathrm{MHz}\right): \delta 194.7,162.8,138.2,128.6,126.3,95.2,44.6,36.7,35.4,28.6,18.6$.

4-Butylamino-pent-3-en-2-one (7). ${ }^{4 \mathrm{j}} \mathrm{IR}\left(\mathrm{cm}^{-1}\right): v_{\text {máx }} 3583,2960,2933,1610,1576,1299,1021$, 739. ${ }^{1} \mathrm{H}$ NMR $\left(\mathrm{CDCl}_{3}, 300 \mathrm{MHz}\right): \delta 10.87$ (br s, 1H), 4.95 (s, 1H), 3.25 (q, 2H, J= $\left.7.3 \mathrm{~Hz}\right), 1.99$ $(\mathrm{s}, 3 \mathrm{H}), 1.92(\mathrm{~s}, 3 \mathrm{H}), 1.60-1.55(\mathrm{~m}, 2 \mathrm{H}), 1.45-1.42(\mathrm{~m}, 2 \mathrm{H}), 0.94(\mathrm{t}, 3 \mathrm{H}, J=7.3 \mathrm{~Hz}) .{ }^{13} \mathrm{C}$ NMR $\left(\mathrm{CDCl}_{3}, 75 \mathrm{MHz}\right): \delta$ 194.4, 163.4, 94.9, 42.6, 32.0, 28.4, 19.9, 18.7, 13.6.

Ethyl-3-(2-aminoethylamino)-but-2-enoate (8). ${ }^{4 \mathrm{n}}$ IR $\left(\mathrm{cm}^{-1}\right): v_{\text {máx }}$ 3296, 2983, 2952, 1650, 1605, 1287, 1020, 726. ${ }^{1} \mathrm{H} \mathrm{NMR}\left(\mathrm{CDCl}_{3}, 300 \mathrm{MHz}\right): 8.64$ (br s, 2H), 4.48 (s, 2H), 4.09 (q, 4H, $J=7.0 \mathrm{~Hz}), 3.37-3.34(\mathrm{~m}, 4 \mathrm{H}), 1.91(\mathrm{~s}, 6 \mathrm{H}), 1.24(\mathrm{t}, 6 \mathrm{H}, J=7.0 \mathrm{~Hz}) .{ }^{13} \mathrm{C} \mathrm{NMR}\left(\mathrm{CDCl}_{3}, 75\right.$ $\mathrm{MHz}): \delta 170.8,161.6,83.7,58.7,44.0,19.5,14.8$.

4-(2-Aminoethylamino)-pent-3-en-2-one (9). ${ }^{4 \mathrm{j}} \mathrm{Mp}, 111-113{ }^{\circ} \mathrm{C}$ (Lit. ${ }^{4 \mathrm{j}} \mathrm{mp}, 112-114{ }^{\circ} \mathrm{C}$ ); IR $\left(\mathrm{cm}^{-1}\right): v_{\text {máx }} 3451,3169,3080,2948,2915,1611,1578,1287,1021,739 .{ }^{1} \mathrm{H} \mathrm{NMR}\left(\mathrm{CDCl}_{3}, 300\right.$ 
MHz): 10.98 (br s, 2H), 4.98 (s, 2H), 3.40 (d, 2H), 1.99 (s, 6H), $1.88(\mathrm{~s}, 6 \mathrm{H}) .{ }^{13} \mathrm{C} \mathrm{NMR}\left(\mathrm{CDCl}_{3}\right.$, $75 \mathrm{MHz}): \delta 195.2,162.6,96.0,43.3,28.7,18.5$.

3-Phenylamino-cyclohex-2-enone (10). ${ }^{4 \mathrm{~d}} \mathrm{Mp}, 178-180{ }^{\circ} \mathrm{C}$; $\left(\mathrm{Lit}^{4 \mathrm{~d}} \mathrm{mp}, 178-180{ }^{\circ} \mathrm{C}\right)$; IR $\left(\mathrm{cm}^{-1}\right)$ : $v_{\text {máx }} 3264,2943,2891,1592,1571,1530,1451,1244,1183,704 ;{ }^{1} \mathrm{H}$ NMR $\left(\mathrm{CDCl}_{3}, 300 \mathrm{MHz}\right): \delta$ 7.50-7.10 (m, 5H), 6.15 (brs, 1H), $5.50(\mathrm{~s}, 1 \mathrm{H}), 2.45(\mathrm{t}, 2 \mathrm{H}), 2.33(\mathrm{t}, 2 \mathrm{H}), 1.99(\mathrm{qt}, 2 \mathrm{H}) .{ }^{13} \mathrm{C} \mathrm{NMR}$ $\left(\mathrm{CDCl}_{3} 75 \mathrm{MHz}\right): \delta 198.4,163.6,138.2,128.9,125.1,123.7,98.6,36.3,29.3,21.7$.

3-(2-Methyl-phenylamino)-cyclohex-2-enone (11). IR $\left(\mathrm{cm}^{-1}\right): \mathrm{mp}, 132-134{ }^{\circ} \mathrm{C} ; v_{\text {máx }} 3223$, 3024, 2940, 1567, 1523, 1463, 1246, 1184, 666. ${ }^{1} \mathrm{H}$ NMR $\left(\mathrm{CDCl}_{3}, 300 \mathrm{MHz}\right): \delta$ 7.23-7.14 (m, 4H), 6.12 (br s, 1H), $5.06(\mathrm{~s}, 1 \mathrm{H}), 2.52(\mathrm{t}, 2 \mathrm{H}, J=6.5 \mathrm{~Hz}), 2.34(\mathrm{t}, 2 \mathrm{H}, J=6.5 \mathrm{~Hz}), 2.21(\mathrm{~s}, 3 \mathrm{H})$, 2.01 (qt, $2 \mathrm{H}, J=6.5 \mathrm{~Hz}) .{ }^{13} \mathrm{C} \mathrm{NMR}\left(\mathrm{CDCl}_{3}, 75 \mathrm{MHz}\right): \delta 197.9,164.3,144.4,136.1,130.9$, 126.7, 118.4, 114.8, 98.6, 36.2, 29.0, 21.8, 17.6.

3-(2-Methoxy-phenylamino)-cyclohex-2-enone (12). ${ }^{11} \mathrm{Mp}, 127-128{ }^{\circ} \mathrm{C}$ (Lit. ${ }^{11} \mathrm{mp}, 129$ $\left.131{ }^{\circ} \mathrm{C}\right)$; IR $\left(\mathrm{cm}^{-1}\right): v_{\text {máx }} 3238,2946,2835,1594,1567,1527,1467,1258,1181,666 .{ }^{1} \mathrm{H}$ NMR $\left(\mathrm{CDCl}_{3}, 300 \mathrm{MHz}\right): \delta 7.32(\mathrm{~d}, 1 \mathrm{H}, J=8,0 \mathrm{~Hz}), 7.10-7.05(\mathrm{~m}, 1 \mathrm{H}), 6.95-6.85(\mathrm{~m}, 2 \mathrm{H}), 6.27(\mathrm{~s}$, $1 \mathrm{H}), 5.73(\mathrm{~s}, 1 \mathrm{H}), 3.84(\mathrm{~s}, 3 \mathrm{H}), 2.52(\mathrm{t}, 2 \mathrm{H}, J=6.6 \mathrm{~Hz}), 2.37$ (t, 2H, $J=6.2 \mathrm{~Hz}), 2.06-2.01(\mathrm{~m}$, 2H). ${ }^{13} \mathrm{C} \mathrm{NMR}\left(\mathrm{CDCl}_{3}, 75 \mathrm{MHz}\right): \delta 198.5,161.7,151.1,127.0,125.4,123.3,120.3,110.9,99.6$, 55.4, 36.2, 29.6, 21.6.

3-(3-Methoxy-phenylamino)-cyclohex-2-enone (13). ${ }^{11} \mathrm{Mp}, 126-128{ }^{\circ} \mathrm{C}$ (Lit. ${ }^{11} \mathrm{mp}, 122,5$ $\left.124^{\circ} \mathrm{C}\right)$; IR $\left(\mathrm{cm}^{-1}\right): v_{\operatorname{máx}} 3278,2995,2835,1615,1583,1541,1494,1296,1195,680 .{ }^{1} \mathrm{H}$ NMR $\left(\mathrm{CDCl}_{3}, 300 \mathrm{MHz}\right): \delta$ 7.25-7.20 (m, 2H), 6.95 (brs, $\left.1 \mathrm{H}\right), 6.80-6.65(\mathrm{~m}, 2 \mathrm{H}), 5.59(\mathrm{~s}, 1 \mathrm{H}), 3.75(\mathrm{~s}$, $3 \mathrm{H}), 2.49(\mathrm{t}, 2 \mathrm{H}, J=6.3 \mathrm{~Hz}), 2.33(\mathrm{t}, 2 \mathrm{H}, J=6.3 \mathrm{~Hz}), 2.03-1.95(\mathrm{~m}, 2 \mathrm{H}) .{ }^{13} \mathrm{C} \mathrm{NMR}\left(\mathrm{CDCl}_{3}, 75\right.$ $\mathrm{MHz}): \delta 198.6,163.6,160.1,139.3,129.8,116.0,110.7,109.6,99.0,55.2,36.2,29.3,21.6$.

\section{Acknowledgements}

We thank the Instituto de Química, Universidade de Brasília, FINATEC, CAPES and CNPq, for financial support, FINEP-CT INFRA n ${ }^{\circ}$ 0970/01, and Eleno Gonçalves from Automação Analítica for providing the microwave reactor.

\section{References and Notes}

1. (a) Loupy, A. Microwave in Organic Synthesis. Ed.; Wiley-VCH: Weinheim, 2002. (b) Tierney, J. P., Lindström, P. Microwave Assisted Organic Synthesis. Eds.; Blackwell, Oxford, 2005. (c) Kappe, C. O. Angew. Chem. Int. Ed. 2004, 43, 6250. (d) Lidström, P., Tierney, J. P., Wathey, B., Westman, J. Tetrahedron 2001, 57, 9225. (e) Kappe, C. O. Angew. Chem. Int. Ed. 2004, 43, 6250. (f) Hayes, B. L. Aldrichimica Acta 2004, 37, 66.

2. (a) Varma, R. S. Pure Appl. Chem. 2001, 73, 193. (b) Varma, R. S. Indian J. Chem., Sec. B 2006, 45B, 2305. 
3. Seijas, J. A., Vázquez-Tato, M. P. Chimica Oggi 2007, 25, 20.

4. (a) Braibante, M. E. F., Braibante, H. T. S., Rosso, G. B., Oriques, D. A. J. Braz. Chem. Soc. 2003, 14, 994. (b) Braibante, H. T. S., Braibante, M. E. F., Morel, A. F., Costa. C. C., Lima, M. G. J. Braz. Chem. Soc. 2006, 17, 184. (c) Das, B., Venkteswarlu, K., Majhi, A., Reddy, M. R., Reddy, K. N., Rao, Y. K., Ravikumar, K., Sridhar, B. J. Mol. Catal. A: Chem. 2006, 246, 276. (d) Gholap, A. R., Chakor, N. S., Daniel, T., Lahoti, R. J., Srinivasan, K. V. J. Mol. Catal. A: Chem. 2006, 245, 37. (e) Arcadi, A., Bianchi, G., Giuseppe, D. S., Marinelli, F. Green Chem. 2003, 5, 64. (f) Giuseppe, B., Marcella, B., Manuela, L., Enrico, M., Paolo, M., Letizia, S. Synlett 2004, 239. (g) Gao, Y., Zhang, Q., Xu, J. Synth. Commun. 2004, 34, 909. (h) Khosropour, R., Khodaei, M. K., Kookhazadeh, M. Tetrahedron Lett. 2004, 45, 1725. (i) Lenin, R., Raju, R. M. Arkivoc 2007, 204. (j) Zhang, Z.-H., Yin, L., Wang, Y.-M. Adv. Synth. Catal. 2006, 348, 184. (k) Zhang, Z.-H., Hu, J.-Y. J. Braz. Chem. Soc. 2006, 17, 1447. (1) Zhang, Z.-H., Song, L.-M. J. Chem. Res. 2005, 817. (m) Khodaei, M. M., Khosropour, A. R., Kookhazadeh, M. Can. J. Chem. 2005, 83, 209. (n) Stefani, H. A., Costa, I. M., Silva, D. O. Synthesis 2000, 1526. (o) Lee, D. H., Park, S.-E., Cho, K., Kim, Y., Athar, T., Lee, I.-M. Tetrahedron Lett. 2007, 48, 8281.

5. (a) Ferraz, H. M. C., Gonçalo, E. R. S. Quím. Nova 2007, 30, 957. (b) Ferraz, H. M. C., Pereira, F. L. C. Quím. Nova 2004, 27, 89. (c) Rappoport, Z. The Chemistry of Enamines. Ed.; John Wiley \& Sons: New York, Part 1, 1994.

6. Stanovnik, B., Svete, J. Chem. Rev. 2004, 104, 2433.

7. Michael, J. P., Koning, C. B., Gravestock, D., Hosken, G. D., Howard, A. S., Krause, R. W. M., Parsons, A. S., Pelly, S. C., Stanbury, T. V. Pure Appl. Chem. 1999, 71, 979.

8. (a) Baraldi, G., Simoni, D., Manfredini, S. Synthesis 1983, 902. (b) Martin, D. F., Janusonis, G. A., Martin, B. B. J. Am. Chem. Soc. 1961, 83, 73.

9. Rechsteiner, B., Texier-Boullet, F., Hamelin, J. Tetrahedron Lett. 1993, 34, 5071.

10. Venkov, A. P., Angelov, P. A. Synthesis 2003, 2221.

11. Iida, H., Yuasa, Y., Kibayashi, C. J. Org. Chem. 1980, 45, 2938. 